Anderson, E. S. \& Felix, A. (1953). J. gen. Microbiol. 8, 408-420.

\title{
'Degraded Vi Strains' and Variation in Vi-phage II of Salmonella typhi
}

\author{
By E. S. ANDERSON AND A. FELIX \\ Central Enteric Reference Laboratory and Bureau, Public Health Laboratory \\ Service, Colindale Avenue, London, N.W. 9
}

SUMMARY: The propagation of Vi-phage II on 'degraded Vi strains' of Salmonella typhi did not yield phage preparations bearing any trace of the specificity of the original Vi type from which the degraded strains had sprung.

Ten different standard Vi-typing phages were grown on a selection of degraded Vi strains. The phages could be separated into two groups: six underwent a change which appeared to be a phenotypic modification, and four were propagated unchanged. It seems probable, however, that all adapted Vi-typing preparations are phenotypic modifications of Vi-phage II.

In the attack of Vi-phage II on the typhoid bacillus the Vi antigen apparently plays a part only in the adsorption of the phage. The stages of phage growth succeeding adsorption seem to be independent of the Vi antigen, and it is in the regions of the bacterial cell concerned with these stages that Vi-type specificity seems to reside.

The typing of strains of Salmonella typhi by means of adapted Vi bacteriophages, first introduced by Craigie \& Yen in 1938, has established itself as the most reliable guide in the epidemiological link-up of cases, carriers and vehicles of infection in typhoid fever. The standardized technique recommended by Craigie \& Felix (1947) has been adopted as the provisional international standard method. It is based on the employment of standard Vi-phage preparations and the corresponding Vi-type strains that are distributed by the International Reference Laboratory for enteric phage typing (London). The revised typing scheme of Sal. typhi suggested by Craigie \& Felix (1947) contained twenty-four types and subtypes. Five new types were added by workers in different countries during the next three years (International Committee for Enteric Phage Typing, 1950), and the extended scheme now in use, which was published in full in a preceding paper (Felix \& Anderson, $1951 a$ ), comprises twenty-nine recognized types and subtypes.

Since the early work of Craigie \& Yen (1938) it has been recognized that the chief difficulties encountered in the Vi-phage typing of typhoid strains are those arising from the 'degradation' of cultures. Various aspects of this phenomenon were subsequently discussed by Craigie (1942) and by Craigie \& Felix (1947). More recently Felix \& Anderson (1951 a) described a striking instance of this complication met with in routine phage-typing.

The experiments recorded in the present paper were designed primarily to determine whether it would be possible to trace the specific Vi type from which a 'degraded' variant had arisen. It was hoped that the experiments might also throw some light on the mechanisms involved in the degradation of cultures of the specific Vi types of Sal. typhi and in the adaptation of 
Craigie \& Yen's Vi-phage II to the various Vi types. A preliminary note on the latter subject has already been published (Anderson \& Felix, 1952).

'Untypable Vi strains' and 'degraded Vi strains'. A strain of Sal. typhi which contains the full complement of $\mathrm{Vi}$ antigen may be untypable for a number of reasons. First, it may be insensitive to Vi-phage II, either because it belongs to a type for which a specifically adapted phage has not yet been prepared, or because it is totally resistant to the action of Vi-phage II. The term 'untypable Vi strain' is now reserved for such strains and is no longer applied to all Vi-positive cultures which cannot be typed with Vi-phage II (see Craigie \& Felix, 1947; Felix \& Anderson, 1951 a). Secondly, the strain may be a 'degraded Vi strain'. Degradation consists of the appearance of cross-reactions with heterologous typing phages so that the original specificity of the strain becomes obscured. In its most advanced stage the change results in the conversion of a specific type into Type $\mathbf{A}$, the latter giving confluent lysis with all the typing phages. Tests of single colonies picked from a plating of a degraded culture may show that some colonies still give the specific phage reactions of the original type while others give the degraded reaction. Degraded colonies picked in this way may vary amongst themselves in the degree of cross-reactions. It is apparent then that the process of degradation may take place in a series of progressive stages of which the specific type occupies one extreme and Type $\mathbf{A}$ the other.

According to Felix (1951), only $3.0 \%$ of strains of the typhoid bacillus examined in the British Isles between 1942 and 1949 were 'degraded $\mathrm{Vi}$ strains' at the time the cultures were first subjected to the bacteriophage tests.

Craigie \& Yen (1938) suggested that it might be possible by the adaptation of Vi-phage II to degraded variants of Sal. typhi to trace the specific types from which the variants had sprung. They called this method 'indirect typing'.

\section{EXPERIMENTAL METHODS}

Media

Tryptic digest broth was used throughout as the nutrient basis for media. When used as broth for the propagation of phages, for the growth of organisms alone, and for the preparation of dilutions of phages, $1 \%$ peptone was incorporated. The solid medium employed was tryptic digest broth with the addition of $1.3 \%$ New Zealand powdered agar but without peptone.

\section{Preparation and testing of adapted phages}

The standard typing phages used were titrated on the various strains selected for test. The plates were incubated overnight at $38 \cdot 5^{\circ}$, and single plaques with a little of the surrounding culture were cut from those plates which showed discrete plaques, transferred to tubes containing $3 \mathrm{ml}$. of tryptic digest broth and incubated at $38.5^{\circ}$ for as long as lysis continued. The average period of growth was $7 \mathrm{hr}$. When no apparent lysis took place and the turbidity of the tubes containing phage and culture increased at the same rate as that of the control tube containing culture without phage, incubation 
for the full $7 \mathrm{hr}$. often resulted in loss of titre in the phages. In such cases the optimal incubation time for the production of phages of reasonably high titre was between 5 and $6 \mathrm{hr}$.

The newly grown phages were heated at $57^{\circ}$ for $40 \mathrm{~min}$. to kill the typhoid bacilli, centrifuged, and the supernatant fluid was pipetted off. Each phage preparation so obtained was titrated on the strain on which it had been grown and on the original Type A strain of Craigie \& Yen (1938). This titration enabled suitable dilutions to be selected for test on all the Vi-type strains of the typhoid bacillus, in order to identify the type specificity of the new phages.

\section{Table 1. Example of preliminary titration of phage preparations}

Phage preparations grown from single plaques

\begin{tabular}{|c|c|c|c|c|}
\hline \multirow[b]{2}{*}{ Culture } & \multirow{2}{*}{$\begin{array}{l}\text { Phage } \\
\text { dilutions }\end{array}$} & \multicolumn{3}{|c|}{ Phage E 1 /strain 9} \\
\hline & & Plaque 1 & Plaque $\mathbf{2}$ & Plaque 3 \\
\hline Type A & $\begin{array}{l}10^{-4} \\
10^{-5} \\
10^{-6} \\
10^{-7} \\
10^{-8}\end{array}$ & $\begin{array}{l}\text { CL } \\
++n \\
+n \\
1 n \\
1 n\end{array}$ & $\begin{array}{c}\text { CL } \\
+++n \\
+n \\
\pm n \\
-\end{array}$ & $\begin{array}{c}\text { CL } \\
\text { CL } \\
++n \\
+n \\
-\end{array}$ \\
\hline $\begin{array}{l}\text { Degraded strain } 9 \text { (derived } \\
\text { from Type D5) }\end{array}$ & $\begin{array}{l}10^{-4} \\
10^{-6} \\
10^{-6} \\
10^{-7} \\
10^{-8}\end{array}$ & $\begin{array}{l}\text { CL } \\
+n \\
\pm n \\
- \\
-\end{array}$ & $\begin{array}{c}\text { CL } \\
+++n \\
+n \\
\pm n \\
1 n\end{array}$ & $\begin{array}{c}\text { CL } \\
\text { CL } \\
++n \\
+n \\
-\end{array}$ \\
\hline Sal. typhi, strain 0901 & $10^{-2}$ & - & - & - \\
\hline $\begin{array}{l}\text { Sal. typhimurium, } \\
\text { strain } 3040\end{array}$ & $10^{-2}$ & - & - & - \\
\hline
\end{tabular}

$\mathrm{CL}=$ confluent lysis with standard loopful of test dilution of phage; $-=$ no plaques; $\pm=5-10$ plaques;,,$++++++=$ increasingly numerous plaques; $1 n=$ one normal plaque (diameter $1 \mathrm{~mm}$. or more).

Table 1 gives an example of the preliminary titration of three phage preparations grown from selected single plaques. The table shows that each phage preparation was active to about the same titre on Type $A$ and on the strain on which it had been propagated (degraded strain 9). In addition, two control strains were included in these tests as a routine procedure-Sal. typhi strain 0901 and $S a l$. typhimurium strain 3040-in order to detect any contaminant $O$ phages that might have been picked up accidentally during the process of propagation. These two strains, known from earlier work of one of us (A.F.) to be permanent Vi-negative variants, have been employed as reagents for the detection of $O$ phages throughout the work on paratyphoid $\mathbf{B}$ Vi-phage typing (Felix \& Callow, 1943, 1951). In the course of the present investigation it was found that the newly grown phage preparations invariably contained only the pure Vi-phage in the dilutions tested.

Phages were designated as fractions of which the numerator indicated the parent phage and the denominator the strain on which the phage had been propagated. For example, phage E $1 /$ strain 9 indicated a phage resulting from the propagation of the standard typing phage $\mathbf{E} 1$ on strain 9 (see Table 1 ). 
If the preliminary titration showed that the propagated phages were of reasonably high titre, i.e. giving discrete plaques at a dilution of $10^{-7}$ to $10^{-8}$ on both Type $\mathbf{A}$ and the strain used for propagation, a full test was set up of selected dilutions of the preparation against the complete array of Vi-type strains of the typhoid bacillus (see Table 2). The dilutions used in these tests were: the highest dilution that produced confluent lysis on the propagating strain and on Type A, and a concentration one hundred times as strong as this. In this way the attack spectra of the newly propagated phages were determined, and their Vi-types identified.

Table 2. Example of cross-tests for type specificity of phage preparations

Dilutions of phage preparations grown from single plaques

Phage E1/strain 9

\begin{tabular}{|c|c|c|c|c|c|c|c|c|}
\hline \multirow{2}{*}{$\begin{array}{l}\text { Vi-type } \\
\text { strains }\end{array}$} & \multicolumn{2}{|c|}{ Plaque 1} & \multicolumn{2}{|c|}{ Plaque 2} & \multicolumn{2}{|c|}{ Plaque 3} & \multicolumn{2}{|c|}{$\begin{array}{l}\text { Control parent } \\
\text { Phage E } 1\end{array}$} \\
\hline & $10^{-2}$ & $10^{-4}$ & $10^{-2}$ & $10^{-4}$ & $10^{-2}$ & $10^{-4}$ & $10^{-3}$ & $0.3 \times 10^{-}$ \\
\hline $\mathbf{A}$ & CL & SCL & CL & CL & CL & CL & CL & SCL \\
\hline C & $\pm m$ & - & $+++m$ & $1 m$ & CL & $7 m$ & $+s$ & - \\
\hline D1 & $4 m$ & - & $\pm m$ & - & $+m$ & - & $\pm m$ & - \\
\hline D2 & - & - & $2 m$ & - & - & - & - & - \\
\hline D4 & $3 m$ & - & - & - & $5 m$ & - & $2 m$ & - \\
\hline D5 & - & - & $\pm m$ & - & $\pm m$ & - & $5 m$ & - \\
\hline D6 & - & - & - & - & - & - & - & - \\
\hline E 1 & $2 m$ & - & $\pm m$ & - & $+m$ & $2 m$ & CL & SCL \\
\hline E2 & - & - & - & - & - & - & $4 n$ & - \\
\hline$F_{1}$ & - & - & - & - & - & - & - & - \\
\hline $\mathrm{F} 2$ & - & - & - & - & - & - & - & - \\
\hline $\mathrm{G}$ & $1 m$ & - & $2 m$ & - & $\pm s$ & - & - & - \\
\hline $\mathbf{H}$ & $+m$ & - & $+ \pm n$ & - & $+\overline{+}+m$ & - & $\pm m$ & - \\
\hline J & - & - & $1 \bar{m}$ & - & $2 m$ & - & - & - \\
\hline K & - & - & - & - & - & - & - & - \\
\hline$L_{1}$ & - & - & $2 m$ & - & $2 m$ & - & - & - \\
\hline L2 & - & - & $1 m$ & - & $\pm m$ & - & - & - \\
\hline$M$ & - & - & $\pm m$ & - & $\overline{3} m$ & - & - & - \\
\hline
\end{tabular}

SCL $=$ semi-confluent lysis: $s=$ small plaques, visible to the naked eye; $m=$ minute plaques, visible only by means of magnifying lens $(\times 10)$. Other symbols as Table 1 .

Table 2 illustrates in abridged form the cross-tests for type specificity of phage preparations. In the experiment recorded in Table 2 none of the three single-plaque phages tested gave the same lytic reactions as the parent phage $\mathrm{E} 1$, but all reacted in a way identical with that of the standard typing phage $A$. The latter has been omitted from the table for the sake of simplicity.

Phage preparations grown from 149 single plaques were examined in the mannor illustrated in Tables 1 and 2. The experiments were divided into two groups.

\section{RESULTS}

Experiments in group 1

In this group of experiments phage $\mathbf{A}$ was propagated on a number of Type A strair:s encountered in the routine work of the laboratory. The strains had been isolat 1 from outbreaks occurring in this country at different times and in 
different localities. Type A is the most prevalent of the typhoid Vi-phage types indigenous to Great Britain; its average percentage distribution during the eight years $1942-9$ was $27 \cdot 3 \%$ (Felix, 1951). As it is believed that many of these strains are descended from specific Vi-phage types which have undergone degradation, these experiments were carried out in order to determine whether any residuum of specificity remained in the strains that could be transmitted to the phages grown on them.

This series can be summarized briefly. All phages resulting from the propagation of phage A on Type A strains were unchanged phage A. From this two possible conclusions could be drawn. Either the Type A strains employed were not descended from specific types or, if they were, the transition to Type $\mathbf{A}$ was accompanied by a loss of the ability to produce phages bearing any specificity for the original type.

\section{Experiments in group 2}

In the second group of experiments ten of the standard typing phages were grown on degraded variants of strains which originally had been found to belong to some of the recognized $\mathrm{Vi}$-phage types. The following phages were used in this series: phages C, D4, D5, D6, E 1, F1, L1, N, O and T. Table 3 lists the eight strains of Sal. typhi employed and also shows which phages were propagated on each strain.

Table 3. List of 'degraded Vi strains' and specific Vi-type phages used in the experiments in group 2

Degraded Vi strains

$\begin{array}{cccl}\begin{array}{c}\text { Original } \\ \begin{array}{c}\text { Vi-phage type } \\ \text { of strain }\end{array}\end{array} & \begin{array}{c}\text { Serial no. } \\ \text { of strain }\end{array} & \begin{array}{c}\text { Vi-phage reaction of } \\ \text { selected 'degraded' } \\ \text { substrains }\end{array} & \begin{array}{c}\text { Standard typing phages propagated } \\ \text { on the degraded substrains }\end{array} \\ \text { D5 } & \mathbf{9} & \text { Type A } & \text { C, D4, D 5, D6, E 1, F 1, L 1, N, O, T } \\ \text { D5 } & 10 & \text { Type A } & \text { D4, D6, F 1, L 1, N, T } \\ \text { N } & 1 & \text { Degraded } & \text { D6, E 1 } \\ \text { N } & 2 & \text { Degraded } & \text { D5, T } \\ \text { N } & 3 & \text { Degraded } & \text { F1, O, N } \\ \text { O } & 7 & \text { Type A } & \text { C, D4, D5, D6, E 1, F 1, L 1, N, O, T } \\ \text { O } & 8 & \text { Type A } & \text { C, D4, D6, F 1, L 1, N, T } \\ \text { T } & 11 & \text { Type A } & \text { C, D4, D 5, D6, E 1, F 1, N, O, T }\end{array}$

Table 3 shows that all the strains had lost the specific Vi-phage reactions of the strains from which they sprang. Those derived from the three Type $\mathbf{N}$ strains (nos. 1, 2 and 3) gave confluent lysis with phages $\mathbf{N}$ and $\mathrm{T}$; semiconfluent lysis or less with phages D1, D2, D4, D6, F1 and G; and crossreactions of varying degree with the other typing phages. All colonies of the former Type $\mathbf{N}$ strains exhibited this partly degraded reaction. The remaining five strains gave the full reaction of Type $\mathbf{A}$.

The strains were plated and single colonies picked and tested with the standard typing phages. Though most of the single colonies of strains which reacted as Type A were fully susceptible to all the typing phages, some showed the less complete degraded reaction. Only lines giving the full cross-reaction 
of Type A were used in subsequent experiments on these cultures. Singlecolony lines were retained for all the experiments in group 2, and the purity of these lines was checked daily by careful colony selection.

In the preliminary titration of the adapted phages used as the starting points of this series, it was found that their titres on each of the degraded cultures giving the Type A reaction were the same as those on the homologous strains for which they were specific: for example, phage $\mathrm{E} 1$ titrated on strain 9 and on Type $\mathbf{E} 1$ gave individual plaques at about $10^{-8}$ on both strains.

The ten specific Vi-typing phages grown on degraded organisms giving the full Type A reaction could be separated into two groups as shown in Table 4. Four of the phages were propagated unchanged and five were changed to phage A. One of the phages (D4) is not included in Table 4. Phage D4 was propagated as phage $\mathrm{D} 1$ on Type A; this will be discussed later.

Table 4. Results of propagation of specific Vi-type phages on Type A cultures derived by degradation from specific Vi-type strains

$\begin{array}{cc}\begin{array}{c}\text { Phages changed } \\ \text { to phage A } \\ \text { on propagation }\end{array} & \begin{array}{c}\text { Phages } \\ \text { propagated } \\ \text { unchanged }\end{array} \\ \text { C } & \text { D5 } \\ \text { E 1 } & \text { D6 } \\ \text { F 1 } & \text { O } \\ \text { L 1 } & \text { N } \\ \text { T } & \end{array}$

The change from the various adapted phages to phage $\mathbf{A}$ occurred uniformly in all of the many plaques cut. The phage $\mathbf{A}$ so produced was indistinguishable from the ancestral phage A, being highly specific for Type A at selected concentrations but containing variant particles able to attack other types; no indication could be found that the phages had propagated in their former type-specific state.

In contrast, the four phages D5, D6, $\mathrm{O}$ and $\mathrm{N}$ were propagated unchanged, and we were unable to change them to phage $\mathbf{A}$ by propagation on organisms giving the full Type A reaction.

Cultures which gave the 'degraded' reaction, i.e. those descended from the three strains of Vi-phage Type $\mathbf{N}$ (see Table 3, strains 1, 2 and 3) were much more difficult to work with than were those reacting as Type A. In the first place the sensitivity of these strains to the Vi-typing phages used in this investigation was relatively low, and individual plaques were extremely small and difficult to find. As we considered it essential to work with pure-line phages this constituted a considerable obstacle to the work. Nevertheless, a few phages were grown by propagating phage $\mathrm{T}$ on one of the degraded Type $\mathrm{N}$ substrains. All the resultant phages conformed to the same pattern, that is, they attacked Types $\mathbf{A}, \mathbf{N}$ and $\mathrm{T}$ with equal ease, a finding which indicated indeterminate specificity in these phages. 


\section{DISCUSSION}

The experiments described in this paper show that it is not possible, by the adaptation of Vi-phage II to a degraded variant, to trace the specific type from which the degraded variant has sprung. This finding is of considerable practical importance and was reported at the Fifth International Congress for Microbiology in Rio de Janeiro (International Committee for Enteric Phage Typing, 1950). 'Degraded' Type A variants are occasionally isolated during the course of typhoid outbreaks (see Craigie \& Felix, 1947; Felix \& Anderson, 1951 a). Their common origin with the specific type responsible for the outbreak cannot be established by Vi-phage typing, and it is therefore necessary to seek other criteria to prove this identity of source. Colonial morphology, nutritional requirements, fermentation reactions and sensitivity to phages unrelated to Vi-phage II were all found to be useful ancillary criteria (Felix \& Anderson, 1951 a). Epidemiological evidence may of course be of help, and workers in this field recognize the fact that the appearance of degraded variants in association with one of the specific Vi-phage types of Sal. typhi is occasionally, though fortunately rarely, to be expected.

The difficulty arising from the degradation process - the inability to connect the variant with its parent strain by the phage-typing method-can be reduced to some extent by making use of the fact that the variants occur in clones in platings of specific types showing the degradation. If, during the primary isolation of the typhoid bacillus, a number of colonies are pooled from platings to form the stock culture, the chances of finding the ancestral specific type as well as its degraded progeny are greatly increased. This is a point of considerable importance, as there is a tendency to send single-colony subcultures to reference laboratories for phage typing. If this procedure is followed after enrichment through tetrathionate or selenite broth the risk of a degraded variant being selected is materially increased. For the preparation of cultures submitted for phage typing the pooling of twelve selected colonies is recommended, preferably from primary platings on selective media such as deoxycholate citrate or Wilson Blair agar. Of course, this is not always possible, as very few colonies of the typhoid bacillus may grow in primary platings. On the other hand, in positive blood cultures, a number of colonies are always available for pooling. Twelve-colony subcultures enable a representative sample of the parent strain to be examined, and the possibility of the picture being complicated by degraded variants is thereby reduced. It may be mentioned also that not only may Vi degradation of the kind described be apparent in single-colony subcultures, but that there is an appreciable risk of selecting a Vi-negative variant which will resist the Vi phage altogether. This point has been emphasized in previous papers (Felix, 1943, 1951). If the organism can be isolated from the patient again a twelve-colony culture will usually reveal its Vi-phage type. But it is not always possible to isolate Sal. typhi from cases of typhoid fever more than once, and if later cultures cannot be obtained the phage type of the infecting strain may never be determined and information of epidemiological value is lost. 
Degradation is associated with the loss of Vi-type specificity, but it is not necessarily associated with a loss of virulence, and Type A strains may show a degree of virulence approaching that of the classical strain Ty 2, which is maximal. An example of this was described recently (Felix \& Anderson, $1951 a)$.

\section{Mechanism of degradation}

The mechanism of degradation can be clarified to some extent by combining information afforded by the present experiments with that resulting from work on the phages carried by various Vi types of the typhoid bacillus. It has been shown (Craigie, 1946; Felix \& Anderson, 1951 b; Anderson, 1951) that Vi-phage type specificity is controlled in a number of types of Sal.typhi by the carriage of bacteriophages by the types concerned. Moreover, these type-determining phages are $\mathrm{O}$ and not $\mathrm{Vi}$ phages, as indicated by their lytic action for the Vinegative strain 0901 (Anderson, 1951; Anderson \& Felix, 1953). Furthermore, the artificial production of new Vi-phage types in the laboratory by the treatment of susceptible types such as Type $\mathbf{A}$ with the type-determining phages may be accompanied by a change in susceptibility to $O$ phages (Felix \& Anderson, 1951 $b$ ); and certain naturally occurring lysogenic Vi-phage types of the typhoid bacillus constantly resist one of the high-titre salmonella $O$ phages described by Felix \& Callow (1943) that has been in routine use in this laboratory for the past twelve years. These facts make it appear probable that the $\mathrm{Vi}$ antigen acts only as the primary receptor for the attachment of Viphage II to the organisms; but, having filled this role, it does not seem to play any further part in the determination of Vi-phage type specificity. This argument is supported by the observations of Craigie (1940) that any Vi-phage type of Sal. typhi will adsorb any adapted Vi-phage II preparation, homologous or not with the type, but will only be lysed if the phage type of the organism and the type specificity of the phage are the same.

These findings suggest that the attack of Vi-phage II on the typhoid bacillus can be divided into two stages. The first is the adsorption of the Vi phage to the $\mathrm{Vi}$ antigen; this stage is specific only in so far as it is dependent on the presence of the $\mathrm{Vi}$ antigen. The second stage, leading to multiplication of the phage, is highly specific and only occurs when the types of the phage and organism are the same. It is not known at what point of the cycle the second stage of attack is blocked when the types of phage and organism are different. However, in view of the fact that phage-type specificity is often dependent on lysogenicity, it seems probable that the block would be an exclusion phenomenon due to the presence in the bacterial cell of the type-determining phage.

Two further points merit consideration. First, it has been demonstrated (Felix \& Anderson, 1951 $b$; Anderson, 1951) that phage-free organisms appearing in cultures the Vi-type determination of which depends on lysogenicity acquire a greater range of susceptibility to the Vi-typing phages immediately the carried phage is lost. Secondly, the treatment of the fully sensitive Type A with type-determining phages produces specific Vi-types with a very much reduced range of susceptibility to the Vi-typing phage prepara- 
tions (Craigie, 1946; Felix \& Anderson, 1951 $b$; Anderson, 1951; Anderson \& Felix, 1953). In these cases, then, the width of the spectrum of sensitivity to Vi-typing phages is controlled by the presence or absence in the organism of certain $\mathrm{O}$ phages. Nicolle, Hamon \& Edlinger (1951) and Hamon \& Nicolle (1951) have published similar observations on Sal. paratyphi B.

For some time before the experiments recorded in this paper were begun (1947), one of us (A.F.) held the view that the process of degradation of specific Vi-phage types was an instance of variation by loss. It was assumed that every Vi-positive strain of Sal. typhi, irrespective of its Vi-phage type, possessed all the specific receptors corresponding to the known adapted $\mathrm{Vi}$ type phages; that freshly isolated bacilli belonging to one of the specific Vi-phage types also possessed a full range of inhibitors which rendered inaccessible those receptors corresponding to the heterologous phages; that the inhibitors were gradually lost on subculture, thereby exposing more and more of the heterologous receptors, until finally all the inhibitors were lost and a culture of Type A emerged. The designation 'degraded Vi strain' thus implied that the process was an instance of variation by loss, but the concept was based on mere speculation. The recent work on type-determining phages, quoted in the preceding paragraphs, suggests that in some of the recognized Vi-phage types of Sal. typhi the change in the direction of Type A occurs in a single step, and is associated with the loss of a single factor, namely, the type-determining phage, which is an $\mathbf{O}$ phage. Although the process of type determination does not take place within the Vi-antigen complex, it appears nevertheless to be convenient and justifiable to continue to use the term 'degraded Vi strain', since the bacteria are Vi forms that have undergone loss variation.

\section{Adaptation of Vi-phage $I I$}

It is now possible to examine the information afforded by the present experiments on the mechanism of adaptation of Vi-phage II to the various Vi-types of Salm. typhi. Throughout the following discussion the terms 'phenotypic variation' and 'host-range mutant' are used in order to interpret the phenomena observed in a way comparable to that in current use by bacteriophage workers.

Craigie \& Yen (1938) suggested at an early stage in this work that the acquisition of type specificity by the adapted Vi-typing preparations was due to the selection by the different strains of Sal. typhi of host-range mutants already present in any phage preparation used for adaptation. Craigie (1940) gave estimates of the frequency of occurrence of host-range mutants corresponding to a number of specific Vi-types. Craigie \& Yen used this concept as the working hypothesis in the selection of their different Vi-type strains of the typhoid bacillus, and it has served a most useful purpose. On the other hand, Felix (1949) found it difficult to accept this hypothesis on the ground that it necessitates the assumption that Vi-phage II and Sal. typhi, two apparently distinct biological entities, have evolved two exactly corresponding sets of mutants. He argued that the observed phenomena could be explained with less strain on the imagination on the basis that phage was a self-repro- 
ducing unit of endogenous origin, forming part of the genetic make-up of the bacterium.

However, the experiments described in this paper show that the 'hostrange mutant' conception is not entirely necessary for the explanation of Vi-phage II adaptation. Table 4 shows that, of ten adapted phages employed, five changed to phage $\mathbf{A}$ on propagation on Type $\mathbf{A}$ strains. It has been mentioned in the preceding section that, in the preliminary titration of the original adapted phage preparations, it was found that their titres on the homologous specific type strains were the same as those on the Type A cultures of the degraded strains on which it was intended to propagate the phages. From this it could be concluded that every particle of each phage preparation was equally active both on the homologous and the Type A strains. All plaques cut from titrations on Type A strains of each of the five phages in the left-hand column of Table 4, and propagated in the manner described earlier, yielded phage $\mathbf{A}$. This newly grown phage $\mathbf{A}$ was indistinguishable from the ancestral phage A from which most of the standard typing phages are descended. As no indication could be found that the phage had propagated as the former type, it was concluded that the change from the former type (i.e. C, E1, F1, etc.) to phage $\mathbf{A}$ had occurred in a single step commencing with the particle initiating each plaque which had been cut. This $100 \%$ reversion of adapted phages to phage A suggests that, whatever is responsible for the production by certain particles of adapted phages with a newly acquired host spectrum, the process is not one of selection of host-range mutants but of particles susceptible to a modification of host spectrum without genetic alteration. The change is maintained only as long as the adapted phages do not multiply or the bacterial host on which they are allowed to multiply belongs to the same Vi-phage type as that used for the last adaptation. On the other hand, propagation on Type $\mathbf{A}$ changes all particles to phage $\mathbf{A}$ in a single step. The adaptation may thus be described as a phenotypic variation.

It is not clear from these experiments whether the initiation of plaques during the adaptation of phage $\mathbf{A}$ to the various Vi-phage types of the typhoid bacillus results from the selection of particles possessing this phenotypic plasticity, or of organisms metabolically different from the general population that are able to initiate the change in any particle. The fact that the number of plaques produced by phage $\mathbf{A}$ on a heterologous type is proportional to the concentration of phage suggests that the former hypothesis is more likely to be correct.

In contrast to these findings are those with phages D5, D6, $\mathrm{O}$ and $\mathrm{N}$ (see Table 4) which propagated unchanged. In the course of many experiments with these phages grown on Type A cultures we were unable to change them to phage $\mathbf{A}$, and it was provisionally concluded that they were host-range mutants with genetic continuity. That a phage behaving as a host-range mutant may nevertheless show phenotypic variation is apparent from the change of phage D4, to phage D1 on Type A strains. Phage D4 was first prepared by adapting phage A to Type D1 (Craigie \& Yen, 1938) and then adapting the resulting phage D1 to Type D4 (Felix, 1943). Phage D1 could 
thus be regarded as a selected host-range mutant of phage $\mathrm{A}$ and phage $\mathrm{D} 4$ as a phenotypic variation of phage D1.

Phages grown on organisms giving the partly degraded reaction were few in number because of technical difficulties which have been indicated earlier. The only useful phages in this group were derived from the propagation of phage $\mathbf{T}$ on degraded Type $\mathbf{N}$ strains. The phages so obtained, as has already been pointed out, could not be assigned to one of the recognized Vi-types because they showed lytic activities of equal strength for Type A, Type T and Type N. The interpretation of this lack of specificity was something of a problem until it was realized that if one considered phage $\mathbf{T}$ to be a phenotypic variation of Vi-phage II, contact of such a phage with a strain lacking the distinctive characters of one of the specific Vi-types might impress on it a further phenotypic variation, resulting in a phage that could not be identified by testing on the recognized specific Vi-types of Sal. typhi. This change, therefore, appears to be the same in principle as the others which have been interpreted as phenotypic variations, and it is interesting to note that phage $\mathbf{T}$ belongs to this group when tested on Type A cultures.

In spite of the fact that only six of the ten adapted phages used in these experiments changed their type when propagated on Type A cultures derived from degraded Vi strains, we are inclined to believe that the whole process of adaptation of Vi-phage II to the various Vi-types of the typhoid bacillus is one of phenotypic variation. It is possible that we were unsuccessful in changing the remaining four phages to phage $\mathbf{A}$ because the correct organisms were not employed to precipitate the change.

If it is assumed that the acquisition of Vi-type specificity by Vi-phage II is entirely phenotypic in origin, the difficulty of believing that Vi-phage II has independently evolved variants corresponding to all the possible Vi-types of Sal. typhi disappears. It is only necessary to postulate a labile grouping on the bacteriophage that can be modified, during the processes following adsorption, to fit the specific patterns in the various phage types of the organism. Type specificity is thus considered to be inherent in the bacterium only. This hypothesis is clearly applicable to those phages which changed to phage $A$, to the change of phage $\mathrm{D} 4$ to phage $\mathrm{Dl}$, and to the change of phage $\mathrm{T}$ to one of ill-defined specificity; whether it will be possible to prove that the remaining adapted Vi-phages belong essentially to the same category can only be decided by further work.

It is evident that the general rule, laid down by Craigie $\&$ Felix (1947), of using phage $\mathbf{A}$ as the parent phage for adaptation to new typhoid Vi-types in the preparation of the standard typing phages is a sound one. Phage $\mathbf{A}$ is highly specific for Type $\mathbf{A}$, and a new phage descended from it acquires the additional ability to attack only the new type used for adaptation. In contrast, phages descended from a host-range mutant, such as phage D6, may retain in full the ability to attack Type D6 and can then lyse Type A, Type D6 and the type to which they have been newly adapted.

Interest has been aroused recently in the subject of phenotypic variation in phages, and Luria \& Human (1952) and Bertani \& Weigle (1953) have 
published work which is in many respects similar to that described in this paper, although the phages and host organisms were different. The phenotypic variations impressed on Vi-phage II are directed, at least in some instances, by the alterations produced in the typhoid bacillus by apparently unrelated non-Vi-phages. Weigle \& Delbrück (1951) demonstrated the exclusion of a carried phage $(\lambda)$ in ultra-violet induced (Lwoff, Siminovitch \& Kjeldgaard, 1950) Bact. coli K-12 by infection with phage T 5. They argued that, as phage $\lambda$ was already present in the bacterial cells exposed to attack by T5, it was probable that the exclusion took place at an intracellular level, and that exclusion by resistance of the bacterial cells to penetration as suggested by Lesley, French, Graham \& Van Rooyen $(1951 a, b)$ could be ruled out. In the resistance of the lysogenic Vi-phage types of Sal. typhi to phage A the position is reversed; the carried phage excludes the externally applied phage, and it is evident that the block is at some stage in the multiplication cycle of the invading phage succeeding adsorption. Thus the altered physico-chemical patterns produced in the organism by lysogenicity are at the same time responsible for the resistance of the lysogenic types to phage $A$, and for the phenotypic modification of a fraction of the Vi-phage II particles into forms which are able to multiply in the bacterial cell; these forms constitute the range of Craigie's adapted Vi-phages.

\section{REFERENCES}

Anderson, E. S. (1951). The significance of Vi-phage types F1 and F2 of Salmonella typhi. J. Hyg., Camb. 49, 458.

Anderson, E. S. \& Fetrx, A. (1952). Variation in Vi-phage II of Salmonella typhi. Nature, Lond. 170, 492.

Anderson, E. S. \& Felix, A. (1953). The Vi-type determining phages carried by Salmonella typhi. J. gen. Microbiol. 9 (in the Press).

Bertani, G. \& Weigle, J. J. (1953). Host-controlled variation in bacterial viruses. J. Bact. 65, 113.

Craigie, J. (1940). Variation of type II Vi-phage. Proc. Third Int. Congr. Microbiol., New York, p. 296.

Craigie, J. (1942). The present status of phage typing of Bact.typhosum. Canad. publ. Hlth J. 33, 41.

Craigie, J. (1946). The significance and applications of bacteriophage in bacteriological and virus research. Bact. Rev. 10, 73.

Craigie, J. \& Felix, A. (1947). Typing of typhoid bacilli with Vi bacteriophage. Lancet, i, 823.

Craigie, J. \& Yen, C. H. (1938). The demonstration of types of B. typhosus by means of preparations of type II Vi-phage. Canad. publ. Hlth J. 29, 448, 484.

Felix, A. (1943). Experiences with typing of typhoid bacilli by means of $\mathrm{Vi}$ bacteriophage. Brit. med. J. i, 435 .

Feurx, A. (1949). The nature of bacteriophages. Proc. Fourth Int. Congr. Microbiol., Copenhagen, p. 355.

Felix, A. (1951). Laboratory control of the enteric fevers. Brit. med. Bull. 7, 153.

Felix, A. \& Anderson, E. S. (1951 a). Bacteriophage, virulence and agglutination tests with a strain of Salmonella typhi of low virulence. J. Hyg., Camb. 49, 349. 
Felix, A. \& Anderson, E. S. (1951 b). Bacteriophages carried by the Vi-phage types of Salmonella typhi. Nature, Lond. 167, 603.

Felix, A. \& Callow, B. R. (1943). Typing of paratyphoid-B bacilli by means of Vi bacteriophage. Brit. med. J. ii, 127.

Felix, A. \& Callow, B. R. (1951). Paratyphoid-B Vi-phage typing. Lancet, ii, 10.

Hamon, Y. \& Nicolle, P. (1951). Recherches sur les facteurs qui conditionnent l'appartenance des bacilles paratyphiques $B$ aux différents types bactériophagiques de Felix et Callow. II. Ann. Inst. Pasteur, 80, 496.

International Committee for Enteric Phage Typing (1950). Proc. Fifth Int. Congr. Microbiol., Rio de Janeiro (to be published).

Lesley, S. M., French, R. C., Graham, A. F. \& Van Rooyen, C. E. (1951 $a, b)$. Studies on the relationship between virus and host cell. II and III. Canad. J. med. Sci. 29, 128, 144.

Luria, S. E. \& Human, M. L. (1952). A nonhereditary, host-induced variation of bacterial viruses. $J$. Bact. 64, 557 .

Lwoff, A., Siminovitch, L. \& KJeldgaARd, N. (1950). Induction de la production de bactériophages chez une bactérie lysogène. Ann. Inst. Pasteur, 79, 815.

Nicolle, P., Hamon, Y. \& Edlinger, E. (1951). Recherches sur les facteurs qui conditionnent l'appartenance des bacilles paratyphiques $\mathbf{B}$ aux différents types bactériophagiques de Felix et Callow. I. Ann. Inst. Pasteur, 80, 479.

Weigle, J. J. \& Delbrück, M. (1951). Mutual exclusion between an infecting phage and a carried phage. J. Bact. 62, 301 .

(Received 3 November 1952) 